\title{
The Role of Cognitive Complaints in the
}

\section{Relationship Between Trait Anxiety, Depressive Symptoms, and Subjective Well-Being and III-Being in Adult Community Volunteers}

\author{
Kuniyoshi Toyoshima $\mathbb{D}^{1, *}$ \\ Masahiko Ichiki ${ }^{2, *}$ \\ Takeshi Inoue (D) ${ }^{2}$ \\ Jiro Masuya ${ }^{2}$ \\ Yota Fujimura ${ }^{2,3}$ \\ Shinji Higashi ${ }^{3,4}$ \\ Ichiro Kusumi (D) \\ 'Department of Psychiatry, Hokkaido \\ University Graduate School of Medicine, \\ Sapporo, 060-8638, Japan; ${ }^{2}$ Department \\ of Psychiatry, Tokyo Medical University, \\ Tokyo, 160-0023, Japan; ${ }^{3}$ Department of \\ Psychiatry, Tokyo Medical University \\ Hachioji Medical Center, Tokyo, 193- \\ 0998, Japan; ${ }^{4}$ Department of Psychiatry, \\ Ibaraki Medical Center, Tokyo Medical \\ University, Ibaraki, 300-0395, Japan
}

*These authors contributed equally to this work

Correspondence: Kuniyoshi Toyoshima Department of Psychiatry, Hokkaido University Graduate School of Medicine, Kita 15, Nishi 7, Sapporo, 060-8638, Japan $\mathrm{Tel}+8|011716| 161$

Fax $+81011706508 \mid$

Email toyoshima@med.hokudai.ac.jp
Purpose: Trait anxiety, depressive symptoms, and cognitive complaints affect mental health. The mediating role of cognitive complaints has been reported recently. However, the mediating effects of cognitive complaints in the relationships between trait anxiety, depressive symptoms, and subjective well-being (SWB) and ill-being (SIB) remain unknown. Therefore, we used path analyses to investigate these mediating effects.

Materials and Methods: A total of 554 adult community volunteers in Japan were studied using the State-Trait Anxiety Inventory (Form Y), Patient Health Questionnaire-9, Cognitive Complaints in Bipolar Disorder Rating Assessment, and Subjective Well-Being Inventory. These assessment tools evaluated trait anxiety, depressive symptoms, cognitive complaints, SWB, and SIB. Path analyses were performed in this study.

Results: Path analyses revealed that there were significant indirect effects, via cognitive complaints, of trait anxiety and depressive symptoms on SIB. However, there were no significant indirect effects of trait anxiety and depressive symptoms on SWB. There were significant indirect effects, via depressive symptoms, of trait anxiety on cognitive complaints, SWB, and SIB.

Conclusion: The role of cognitive complaints may be different between SWB and SIB associated with trait anxiety and depressive symptoms. Evaluating the mediating effect of cognitive complaints may be more useful on SIB than SWB associated with trait anxiety and depressive symptoms. These findings may be useful when considering intervention targets in mental health.

Keywords: trait anxiety, depressive symptoms, cognitive complaints, well-being, ill-being, mediator

\section{Introduction}

The constitution of the World Health Organization defined that "health is a state of complete physical, mental and social well-being and not merely the absence of disease or infirmity," and that subjective well-being (SWB) is an important goal in public health. ${ }^{1}$ SWB refers to the cognitive process of contentment, satisfaction, or happiness, ${ }^{2}$ while subjective ill-being (SIB) refers to the negative psychological constructs. ${ }^{3}$ Although the prevention of SIB is as important as the promotion of SWB in public health, the absence of SIB does not automatically lead to SWB and vice versa. ${ }^{4}$ Hence, the influences of various factors that affect mental health must be studied on both SIB and SWB. ${ }^{5}$ 
Trait anxiety and depressive symptoms constitute a public mental health problem. Trait anxiety is a characteristic predisposition to evaluate stimuli as threatening, avoid anxiety-provoking situations, and show high baseline physiological arousal. ${ }^{6}$ In addition, high trait anxiety is considered a key vulnerability phenotype for stress-induced depression. ${ }^{7}$ A recent meta-analysis suggested that individuals with depression had a significantly higher trait anxiety than those with anxiety disorder. ${ }^{8}$ Hence, trait anxiety and depressive symptoms are closely related. Furthermore, both trait anxiety and depressive symptoms affect cognitive function. High trait anxiety correlates with a functional network dysfunction and reduced neural processing efficiency. ${ }^{9,10}$ Depressive symptoms also correlate with neurocognitive impairments. In individuals with depression, network dysfunction underlies core cognitive and affective abnormalities. ${ }^{11-13}$ Cognitive impairment affects functional impairment. ${ }^{14}$ Hence, cognitive impairment associated with trait anxiety and depressive symptoms constitutes a public mental health problem.

Cognitive impairment can be objective or subjective. Objective cognitive impairments can be evaluated using neurocognitive assessments, while subjective cognitive impairments can be evaluated using a self-administered scale. ${ }^{14}$ Psychosocial functioning correlates with more subjective cognitive function than objective cognitive function, ${ }^{13}$ hence, subjective cognitive function is often evaluated in public health. ${ }^{14}$ Subjective cognitive impairments, namely, cognitive complaints, are important factors which affect quality of life and social function in individuals with and without psychiatric illnesses. ${ }^{13-15}$ In addition, cognitive complaints are affected by various factors, including neurocognitive impairments, depression, and anxiety symptoms. ${ }^{16-18}$ In the general adult population, childhood maltreatment, childhood parental bonding, and affective temperaments also influence cognitive complaints. ${ }^{19-21}$ Recently, the mediating roles of cognitive complaints have been reported. Cognitive complaints mediate the influence of depressive symptoms on quality of life and presenteeism. ${ }^{14,15}$ Furthermore, cognitive complaints mediate the influence of affective temperaments, including anxious temperament, on social function. ${ }^{20}$ However, to the best of our knowledge, the mediating role of cognitive complaints in the relationships between trait anxiety, depressive symptoms, SWB, and SIB remains incompletely studied. Depressive symptoms are a main factor in cognitive complaints, and the "depressive symptom $\rightarrow$ cognitive complaints $\rightarrow$ social function" model was previously reported. ${ }^{14}$ Another previous study suggests the "trait marker (affective temperament) $\rightarrow$ state marker (depressive symptoms) $\rightarrow$ cognitive complaints $\rightarrow$ functional disability" model. ${ }^{20}$ However, to our knowledge, the relationship between cognitive complaints and SWB or SIB has not been fully understood. Furthermore, the relationships between trait anxiety, depressive symptoms, and cognitive complaints have not been fully understood. Hence, the present study aimed to investigate the mediating roles of cognitive complaints using path analyses.

\section{Materials and Methods \\ Participants}

We recruited a total of 597 adult volunteers using convenience sampling from April 2017 to April 2018 in Tokyo, Japan. The present study was part of a larger study in which several assessments were used to investigate the relationship between cognitive impairment and social function. ${ }^{14}$ All subjects provided written informed consent to participate in this study, which was conducted in accordance with the Declaration of Helsinki (World Medical Association, 2013). The Local Ethics Committee of Tokyo Medical University approved the present study (approval number: SH3502). The inclusion criteria were set as follows: aged at least 20 years old, not having serious physical illness, not having organic brain damage, and having the capability to provide agreement to participate in the present research. We used only a self-reported questionnaire in this study; hence, the criteria "not having serious physical illness" and "not having organic brain disorder" were evaluated based on patients' own responses. Furthermore, as this was a convenience population sample, and the criterion "having medical illness" was evaluated using patients' responses to the self-reported questionnaire. This study excluded 43 participants who did not complete the assessments; hence, the data of 554 subjects were analyzed.

\section{Trait Anxiety}

The State-Trait Anxiety Inventory (Form Y) (STAI-Y) evaluates degrees of anxiety. It consists of 40 items with a 4-point Likert scale for each item. ${ }^{22}$ Twenty items were about state anxiety, and another twenty were about trait anxiety. Hence, the STAI-Y scores were calculated separately for state anxiety (ranging from 20 to 80) and trait 
anxiety (ranging from 20 to 80). This study only analyzed the items on trait anxiety that were rated on the following 4-point scale: 1 = almost never, $2=$ sometimes, $3=$ often, and $4=$ almost always. The total score ranged from 20 to 80. The higher the score, the more serious the trait anxiety. This study used the validated Japanese version of STAI-Y. ${ }^{23}$

\section{Depressive Symptoms}

The Patient Health Questionnaire-9 (PHQ-9) evaluates the severity of depressive symptoms and consists of nine questions that are rated on a 4-point scale [ranging from 0 to 3 points]. ${ }^{24}$ The total score (ranging from 0 to 27 points) was evaluated as the PHQ-9 score. The higher the score, the more serious the depressive symptoms. ${ }^{25}$ A Japanese version was validated and used in this study. ${ }^{26}$ A PHQ-9 cut-off score of $\geq 10$ indicates depression in the Japanese version. ${ }^{27}$

\section{Cognitive Complaints}

The Cognitive Complaints in Bipolar Disorder Rating Assessment (COBRA) evaluates cognitive impairments which were perceived in daily living. ${ }^{16}$ It consists of 16 items using a 4-point Likert scale as follows for each item: $0=$ never, $1=$ sometimes, $2=$ often, and $3=$ always. The COBRA had a one-factor structure and showed high internal consistency (Cronbach's alpha $=0.913$ ); therefore, the total score was calculated by summing the scores of each item. ${ }^{16}$ High scores indicate serious cognitive impairments. The Japanese version was used in this study. Its validity and reliability is shown. ${ }^{28}$ The COBRA has also been used for evaluating subjective cognitive impairments in the general adult population. ${ }^{14} \mathrm{~A}$ total score of $\geq 15$ represents moderate-to-severe subjective cognitive dysfunction. $^{29}$

\section{Subjective Well-Being and III-Being}

The Subjective Well-Being Inventory (SUBI) evaluates positive affect (well-being) and negative affect (ill-being), and is composed of 40 items (positive affect $=19$ items, negative affect $=21$ items). ${ }^{30}$ SWB is composed of six subscales, while SIB consists of four subscales. The score for each item is rated on a 3-point Likert scale (from 1 to 3). High scores indicate better states in both SWB (ranging from 19 to 57) and SIB (ranging from 21 to 63). We used the Japanese version whose validity and reliability have been demonstrated, and the Japanese version of SUBI, which is composed of seven factors, namely "General happiness," "Upsetability," "Social support," "Physical illhealth," "Family group support," "Deficiency in social contacts," and "Confidence in coping". ${ }^{31,32}$ This study analyzed both SWB and SIB scores.

\section{Statistical Analysis}

Pearson's correlation analysis using Bonferroni correction was conducted to evaluate the relationships between trait anxiety, depressive symptoms, cognitive complaints, SWB, and SIB. A normal probability plot was used to confirm linearity when multiple regression analyses were conducted. A multiple regression analysis by forced entry was conducted to evaluate the influence of demographic characteristics, trait anxiety, depressive symptoms, and cognitive complaints on SWB and SIB. Our conceptual model was "trait marker (trait anxiety) $\rightarrow$ state marker (depressive symptoms) $\rightarrow$ cognitive complaints $\rightarrow$ SWB and SIB." We aimed to investigate the mediating role of cognitive complaints using a path analysis. The goodnessof-fit index was not used in this study because of the saturation model. The standardized path coefficients were calculated to show the strengths of the effects. Statistical analyses were conducted using STATA/MP 16 (Stata Corp LLC, College Station, TX, USA), except for the path analyses, which were performed using Mplus version 8.4 (Muthén \& Muthén, Los Angeles, CA, USA). For all analyses, $p<0.05$ was judged as statistically significant.

\section{Results \\ Basic Findings}

Table 1 presents the demographic characteristics and clinical assessments of the 554 participants. The range of variables (minimum score-maximum score) of clinical assessments are shown in parentheses as follows: STAI$\mathrm{Y}$ trait anxiety (20-78), PHQ-9 (0-24), COBRA (0-34), SUBI well-being (21-57), and SUBI ill-being (28-63). We analyzed the Cronbach's $\alpha$ values of all the questionnaires and subscales as follows: STAI-trait anxiety (0.66), PHQ-9 (0.85), COBRA (0.91), SWB (0.90), SIB (0.87), "General happiness" (0.85), "Upsetability" (0.81), "Social support" (0.87), "Physical ill-health" (0.65), "Family group support" (0.77), "Deficiency in social contacts" $(0.40)$, and "Confidence in coping" (0.79). Fifty-seven subjects (10.3\%) showed PHQ-9 scores $\geq 10$, evaluated as depression, ${ }^{27}$ and 97 subjects (17.5\%) showed COBRA scores $\geq 15$, evaluated as moderate-to-severe subjective cognitive dysfunction. ${ }^{29}$ The Pearson correlation analysis 
Table I Basic Findings $(\mathrm{N}=554)$

\begin{tabular}{|l|l|l|}
\hline Characteristics & Mean (SD) & $\mathbf{N}(\%)$ \\
\hline Age & $41.2(I I .9)$ & \\
\hline Sex (Male/Female) & & $242(43.7) / 312(56.3)$ \\
\hline Married & & $368(66.4)$ \\
\hline Education (years) & $14.7(1.8)$ & \\
\hline Currently employed & & $544(98.2)$ \\
\hline Psychiatric history & & $58(10.5)$ \\
\hline Current psychiatric & & $22(4.0)$ \\
\hline treatment & & $354(63.9)$ \\
\hline Drinking habit & & $103(18.6)$ \\
\hline Smoking habit & & $\mathbf{N}(\%)$ \\
\hline Clinical assessments & Mean (SD) & \\
\hline STAI-Y trait anxiety & $43.0(10.4)$ & \\
\hline PHQ-9 & $4 . I(4.2)$ & \\
\hline PHQ-9 $\geq$ I0 & & $57(10.3)$ \\
\hline COBRA & $51.5(6.4)$ & \\
\hline COBRA $\geq 15$ & & \\
\hline SUBI well-being & & \\
\hline SUBI ill-being & & \\
\hline
\end{tabular}

Abbreviations: COBRA, Cognitive Complaints in Bipolar Disorder Rating Assessment; PHQ-9, Patient Health Questionnaire-9; STAI-Y, State-Trait Anxiety Inventory Form Y; SUBI, Subjective Well-Being Inventory; SD, Standard deviation.

revealed that trait anxiety significantly and positively correlated with depressive symptoms $(\mathrm{r}=0.64, p<0.001)$ and cognitive complaints $(\mathrm{r}=0.43, p<0.001)$. In addition, depressive symptoms significantly and positively correlated with cognitive complaints $(\mathrm{r}=0.40, p<0.001)$. SWB negatively correlated with trait anxiety $(\mathrm{r}=-0.59$, $p<0.001)$, depressive symptoms $(\mathrm{r}=-0.44, p<0.001)$, and cognitive complaints $(\mathrm{r}=-0.27, p<0.001)$. Furthermore, SIB negatively correlated with trait anxiety ( $\mathrm{r}=-0.67, p<0.001)$, depressive symptoms $(\mathrm{r}=-0.58$, $p<0.001$ ), and cognitive complaints ( $\mathrm{r}=-0.45, p<$ 0.001). The positive correlation between SWB and SIB was statistically significant $(\mathrm{r}=0.47, p<0.001)$.

\section{Multiple Regression Analysis}

Table 2 demonstrates the multiple regression analyses of SUBI well-being and ill-being. The significant positive predictors of SUBI well-being were female sex, married status, and long educational years. The significant negative predictors of SUBI well-being were age, trait anxiety, and depressive symptoms. The only significant positive predictor of SUBI ill-being was age. The significant negative predictors of SUBI ill-being included having a psychiatric history, trait anxiety, depressive symptoms, and cognitive complaints.

\section{Path Analysis of Well-Being}

A path analysis was conducted to investigate the relationships among trait anxiety, depressive symptoms, cognitive complaints, and SWB (Table 3, Figure 1). In the model, the coefficient of determination of well-being was 0.351 , namely, the model explained $35.1 \%$ of SWB.

Regarding direct effects, trait anxiety significantly and directly influenced depressive symptoms, cognitive complaints, and SWB. Depressive symptoms significantly and directly influenced cognitive complaints and SWB. However, cognitive complaints had no significant direct effect on SWB.

Concerning indirect effects, trait anxiety significantly influenced cognitive complaints and SWB via depressive symptoms. However, trait anxiety had no significant indirect effect on SWB via cognitive complaints alone or via both depressive symptoms and cognitive complaints. Depressive symptoms did not significantly influence SWB via cognitive complaints.

In summary, the mediating effects of depressive symptoms were demonstrated in the relationships among trait anxiety, cognitive complaints, and SWB, while the mediating effect of cognitive complaints was not demonstrated.

\section{Path Analysis of III-Being}

A path analysis was performed to evaluate the relationships among trait anxiety, depressive symptoms, cognitive complaints, and SIB (Table 4, Figure 2). In this model, the coefficient of determination of ill-being was 0.507 . This means that the model explained $50.7 \%$ of SIB. Further, all the paths were statistically significant (Table 4).

Regarding direct effects, trait anxiety significantly and directly influenced depressive symptoms, cognitive complaints, and SIB. Depressive symptoms significantly and directly influenced cognitive complaints and SIB. In addition, cognitive complaints significantly and directly influenced SIB.

Regarding indirect effects, trait anxiety significantly influenced cognitive complaints via depressive 


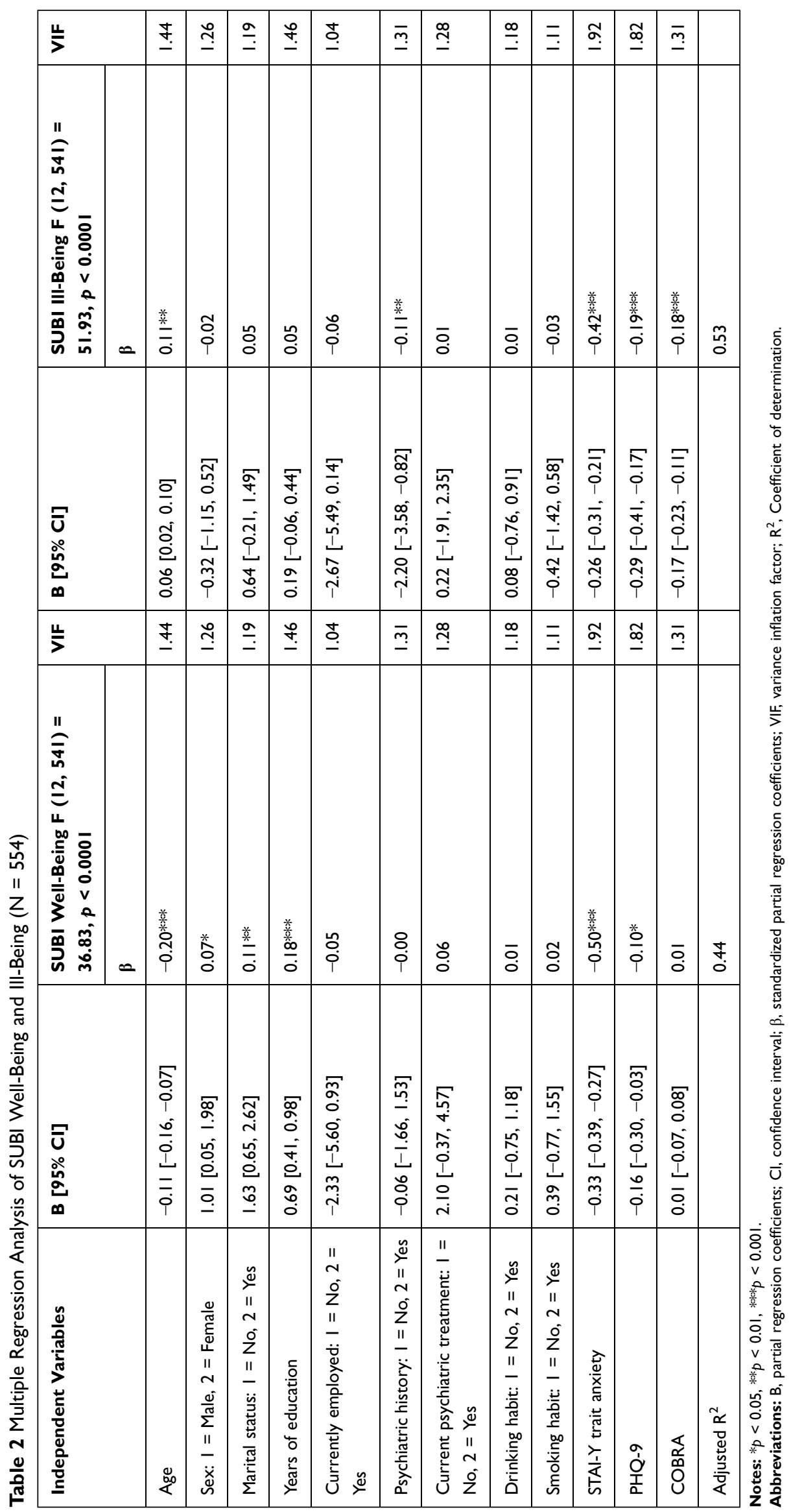


Table 3 Standardized Path Coefficients of Path Analysis of Well-Being for 554 Study Participants

\begin{tabular}{|c|c|c|c|}
\hline \multirow[b]{2}{*}{ From } & \multicolumn{3}{|l|}{ Direct Effect to } \\
\hline & Depressive symptoms & Cognitive complaints & Well-being \\
\hline \multirow{5}{*}{$\begin{array}{l}\text { Trait anxiety } \\
\text { Depressive symptoms } \\
\text { Cognitive complaints }\end{array}$} & $0.642^{* * * *}$ & $0.291 * * *$ & $-0.524 * * *$ \\
\hline & & $0.214 * *$ & $-0.100 *$ \\
\hline & & & 0.000 \\
\hline & \multicolumn{3}{|l|}{ Indirect effect to } \\
\hline & Depressive symptoms & Cognitive complaints & Well-being \\
\hline \multirow{5}{*}{$\begin{array}{l}\text { Trait anxiety } \\
\text { Depressive symptoms }\end{array}$} & & $0.138^{* *}$ (via depressive symptoms) & $-0.064 *$ (via depressive symptoms) \\
\hline & & & 0.000 (via depressive symptoms and cognitive complaints) \\
\hline & & & 0.000 (via cognitive complaints) \\
\hline & & & 0.000 (via cognitive complaints) \\
\hline & \multicolumn{3}{|c|}{ Total indirect effect to } \\
\hline \multirow{4}{*}{$\begin{array}{l}\text { Trait anxiety } \\
\text { Depressive symptoms }\end{array}$} & & $0.138 * *$ & $-0.064 *$ \\
\hline & & & 0.000 \\
\hline & \multicolumn{3}{|l|}{ Total effect to } \\
\hline & Depressive symptoms & Cognitive complaints & Well-being \\
\hline Trait anxiety & $0.642 * * *$ & $0.429 * * *$ & $-0.588 * * *$ \\
\hline Depressive symptoms & & $0.214^{* *}$ & $-0.100 *$ \\
\hline Cognitive complaints & & & 0.000 \\
\hline
\end{tabular}

Notes: $*_{p}<0.05, *_{p}<0.01, *^{*} *_{p}<0.001$.

symptoms and SIB via depressive symptoms alone, cognitive complaints alone, and both depressive symptoms and cognitive complaints. Depressive symptoms significantly influenced SIB via cognitive complaints.

In summary, the mediating effects of depressive symptoms and cognitive complaints were shown in the relationship between trait anxiety and SIB.

\section{Discussion}

The present study suggests that cognitive complaints mediate the influence of trait anxiety and depressive symptoms on SIB, while cognitive complaints may not mediate the influence of trait anxiety and depressive symptoms on SWB. Hence, the influence of trait anxiety and depressive symptoms via cognitive complaints may be different between SIB and SWB.

This study used the COBRA scale to evaluate cognitive complaints. The COBRA contains a lot of items on cognitive disturbances in daily life. ${ }^{16}$ Hence, cognitive complaints may have more affinity to SIB, which had a stronger correlation with negative psychological factors than SWB, which correlates with positive psychological factors. ${ }^{2,3}$ Furthermore, SWB is considered more stable than SIB, because personality traits have a stronger influence on SWB than SIB. ${ }^{30}$ Cognitive complaints evaluated by COBRA are known to be affected by mood symptoms even in the general adult population. ${ }^{14,16}$ Hence, in this study, cognitive complaints are considered state-dependent like depressive symptoms, and cognitive complaints might affect SIB stronger than SWB, while trait anxiety might affect SWB stronger than SIB.

A recent study suggested that cognitive complaints correlated with depressive symptoms but not correlated with objective cognitive impairment in older adults. ${ }^{33}$ In addition, individuals with cognitive complaints that did not progress to dementia showed better objective cognitive performance at the baseline than did individuals whose cognitive complaints progressed to dementia. ${ }^{34}$ A previous study reported that subjective memory impairment correlated with negative well-being in a situation in which depressive symptoms were not controlled. ${ }^{35}$ A minor negative effect of the cognitive complaints on well-being was reported, while factors other than cognitive complaints also affect well-being to a considerable extent in the elderly. ${ }^{36}$ Therefore, like in the 


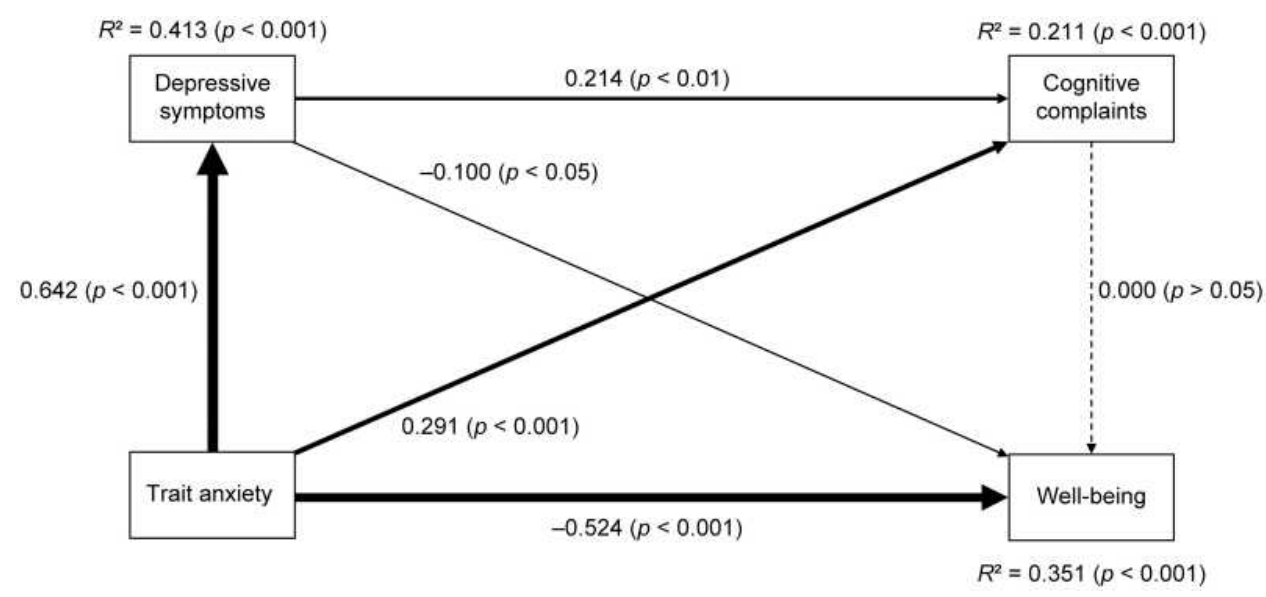

Figure I Relationships among trait anxiety, depressive symptoms, cognitive complaints, and subjective well-being analyzed using a path analysis in 554 participants. The values beside the arrows demonstrate the direct standardized path coefficients. The width of the line demonstrates the strength of the direct effect. Trait anxiety represents the STAI-Y trait anxiety score; depressive symptoms, the PHQ-9 score; cognitive complaints, the COBRA total score; and well-being, the SUBI well-being score.

Abbreviations: COBRA, Cognitive Complaints in Bipolar Disorder Rating Assessment; PHQ-9, Patient Health Questionnaire-9; STAI-Y, State-Trait Anxiety Inventory Form Y; SUBI, Subjective Well-Being Inventory; $\mathrm{R}^{2}$, coefficient of determination.

elderly, the impact of cognitive complaints on well-being could also be minor in adult community volunteers.

In our path models, trait anxiety was used as a personality trait marker, while depressive symptoms and cognitive complaints were used as state markers. Therefore, the effect of trait anxiety might be stronger on SWB. By contrast, the effects of depressive symptoms and cognitive complaints on SIB might be stronger. As a result, the mediating effect of cognitive complaints might be statistically significant only on SIB. Moreover, depressive symptoms mediated the influence of trait anxiety on both SWB and SIB. The direct effect of trait anxiety was stronger on depressive symptoms than on cognitive complaints, and depressive symptoms had stronger direct effects on SWB and SIB than on cognitive complaints. Hence, the mediating effects of depressive symptoms might remain significant in the associations between trait anxiety and SWB and SIB.

To our best knowledge, this is the first study to show that cognitive complaints have different roles in the relationship between trait anxiety, depressive symptoms, and SWB or SIB. In the general adult population, the influences of the factors that affect mental health must be studied on SIB and SWB. ${ }^{5}$ To improve the SWB and SIB which are associated with trait anxiety and depressive symptoms, the mediating role of cognitive complaints should be evaluated on SIB, and the mediating role of depressive symptoms should be evaluated on both SWB and SIB.

This study shows the influence of trait anxiety, depressive symptoms, and cognitive complaints on SWB and SIB. In the general adult population, childhood abuse and affective temperaments influence SWB and SIB. ${ }^{5}$ Further, personality traits mediate the influence of childhood parental bonding on SWB ${ }^{37}$ To address SWB and SIB, it may be useful to evaluate the role of personality traits and the factors that affect personality traits. Careful examination of the factors that influenced personality traits revealed that they also affected depressive symptoms and cognitive complaints. ${ }^{19-21}$ Hence, the relationship among childhood abuse, childhood parental bonding, affective temperaments or personality traits, depressive symptoms, cognitive complaints, and SWB or SIB must be investigated comprehensively in future studies. By doing so, it may contribute to the development of appropriate interventions for SWB and SIB.

Unmet medical needs related to cognitive complaints have attracted attention in recent years. ${ }^{38}$ However, the role of cognitive complaints in mental health, particularly their moderating role, has not been fully understood. The interactions between trait anxiety and cognitive complaints, and between depressive symptoms and cognitive complaints on SWB and SIB, respectively, need to be evaluated in the future. We would like to investigate the moderating effects of cognitive complaints using a hierarchical multiple regression analysis in a future study.

\section{Limitations}

The cross-sectional design could not determine the causal relationships among the factors. Although we suggested the possible mediating role of CCs in our path model, a future longitudinal study is required to evaluate the mediation effect in causality. This research was conducted 
Table 4 Standardized Path Coefficients of Path Analysis of III-Being for 554 Study Participants

\begin{tabular}{|c|c|c|c|}
\hline \multirow[b]{2}{*}{ From } & \multicolumn{3}{|c|}{ Direct Effect to } \\
\hline & $\begin{array}{l}\text { Depressive } \\
\text { symptoms }\end{array}$ & Cognitive complaints & III-being \\
\hline Trait anxiety & $0.642^{* * *}$ & $0.291 * * *$ & $-0.462 * * *$ \\
\hline $\begin{array}{l}\text { Depressive } \\
\text { symptoms }\end{array}$ & & $0.214^{* *}$ & $-0.215^{* * *}$ \\
\hline \multirow[t]{3}{*}{ Cognitive complaints } & & & $-0.164 * * *$ \\
\hline & \multicolumn{3}{|c|}{ Indirect effect to } \\
\hline & $\begin{array}{l}\text { Depressive } \\
\text { symptoms }\end{array}$ & Cognitive complaints & III-being \\
\hline \multirow[t]{3}{*}{ Trait anxiety } & & $\begin{array}{l}0.138^{* *} \text { (via depressive } \\
\text { symptoms) }\end{array}$ & $-0.138^{* * *}$ (via depressive symptoms) \\
\hline & & & $\begin{array}{l}-0.023 * * \text { (via depressive symptoms and cognitive } \\
\text { complaints) }\end{array}$ \\
\hline & & & $-0.048 * *$ (via cognitive complaints) \\
\hline \multirow{2}{*}{$\begin{array}{l}\text { Depressive } \\
\text { symptoms }\end{array}$} & & & $-0.035^{* *}$ (via cognitive complaints) \\
\hline & \multicolumn{3}{|c|}{ Total indirect effect to } \\
\hline Trait anxiety & & $0.138^{* *}$ & $-0.208 * * *$ \\
\hline \multirow{3}{*}{$\begin{array}{l}\text { Depressive } \\
\text { symptoms }\end{array}$} & & & $-0.035 * *$ \\
\hline & \multicolumn{3}{|c|}{ Total effect to } \\
\hline & $\begin{array}{l}\text { Depressive } \\
\text { symptoms }\end{array}$ & Cognitive complaints & III-being \\
\hline Trait anxiety & $0.642^{* * *}$ & $0.429 * * *$ & $-0.670 * * *$ \\
\hline $\begin{array}{l}\text { Depressive } \\
\text { symptoms }\end{array}$ & & $0.214^{* *}$ & $-0.250 * * *$ \\
\hline Cognitive complaints & & & $-0.164 * * *$ \\
\hline
\end{tabular}

Notes: $* * p<0.01, * * * p<0.001$.

in Japan, and thus possibly prevents generalization of our findings to other countries. All participants in this study were adults, which may also prevent generalization of our results to adolescents or children. The heterogeneity of our sample may be a limitation, as both healthy and unhealthy individuals were concurrently recruited and analyzed. In our sample, 57 subjects (10.3\%) showed PHQ-9 scores $\geq$ 10 , evaluated as depression, ${ }^{27}$ and 97 subjects $(17.5 \%)$ showed COBRA scores $\geq 15$, evaluated as moderate-tosevere subjective cognitive dysfunction. ${ }^{29}$ However, the number of individuals who were undergoing psychiatric treatment was $22(4.0 \%)$. Hence, this study included untreated individuals with psychiatric symptoms which could influence the self-assessment questionnaires. Further, the effects of medication at the assessment were not considered. Finally, memory bias could not be corrected because we used self-administered scales in this study.

\section{Conclusion}

The present study suggests that cognitive complaints mediate the influence of trait anxiety and depressive symptoms 


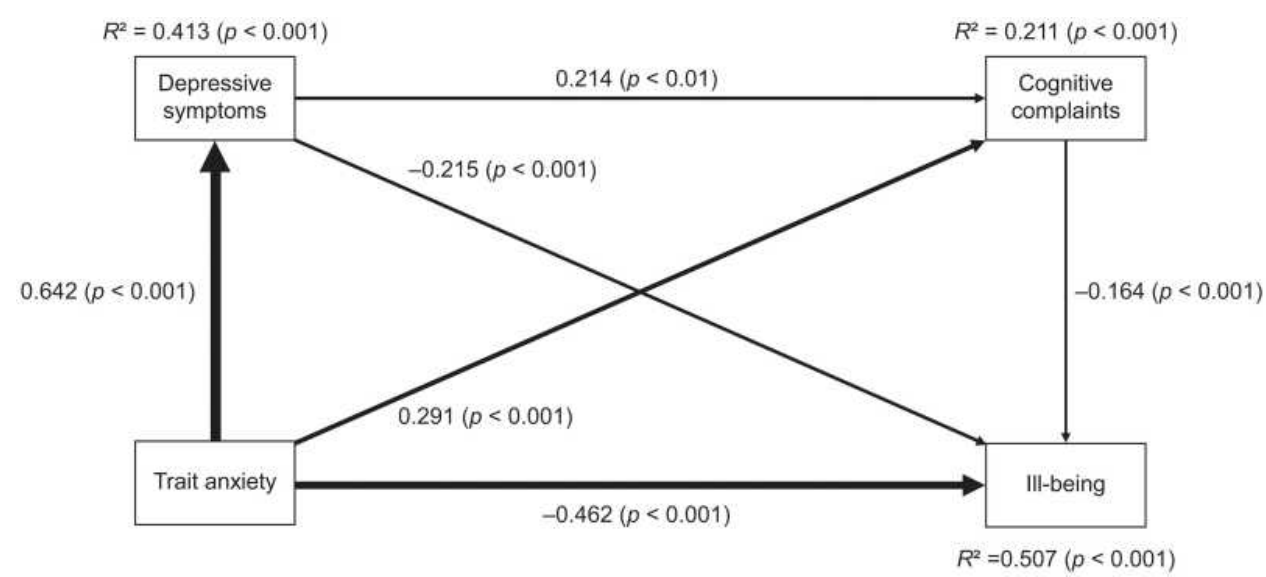

Figure 2 Relationships between trait anxiety, depressive symptoms, cognitive complaints, and subjective ill-being using a path analysis in 554 participants. The values beside the arrows show the direct standardized path coefficients. The width of the line shows the strength of the direct effect. Trait anxiety represents the STAI-Y trait anxiety score; depressive symptoms, the PHQ-9 score; cognitive complaints, the COBRA total score; and ill-being, the SUBI well-being score.

Abbreviations: COBRA, Cognitive Complaints in Bipolar Disorder Rating Assessment; PHQ-9, Patient Health Questionnaire-9; STAI-Y, State-Trait Anxiety Inventory Form Y; SUBI, Subjective Well-Being Inventory; $\mathrm{R}^{2}$, coefficient of determination.

on SIB, while cognitive complaints may not mediate the influence of trait anxiety and depressive symptoms on SWB. Hence, the influence of trait anxiety and depressive symptoms via cognitive complaints may be different between SIB and SWB. To improve SWB and SIB, which are associated with trait anxiety and depressive symptoms, the mediating role of cognitive complaints may need to be evaluated on SIB, and the mediating role of depressive symptoms may need to be evaluated on both SWB and SIB. Evaluating those mediating roles may be useful in considering intervention targets in public mental health.

\section{Data Sharing Statement}

The datasets generated during and/or analyzed during the current study are available from the corresponding author on reasonable request.

\section{Ethics Approval and Informed Consent}

All subjects provided written informed consent to participate in this study, which was conducted in accordance with the Declaration of Helsinki (World Medical Association, 2013). The Local Ethics Committee of Tokyo Medical University approved the present study (approval number: SH3502).

\section{Acknowledgments}

The authors thank Dr. Nobutada Takahashi of Fuji Psychosomatic Rehabilitation Institute Hospital, Dr. Hiroshi Matsuda of Kashiwazaki Kosei Hospital, deceased Dr. Yasuhiko Takita of Maruyamasou Hospital, and
Dr. Yoshihide Takaesu of Izumi Hospital for collecting data. The authors also thank their colleagues at Tokyo Medical University and Hokkaido University. This work was supported by JSPS KAKENHI Grant No. JP20K16662.

\section{Funding}

This work was partly supported by a Grant-in-Aid for Scientific Research (no. 16K10194, to TI) from the Japanese Ministry of Education, Culture, Sports, Science and Technology, Research and Development Grants for Comprehensive Research for Persons with Disabilities from the Japan Agency for Medical Research and Development (no. JP18dk0307060, to TI), SENSHIN Medical Research Foundation (to TI, grant number not available), and JSPS KAKENHI [no. JP20K16662 to KT]. The funding sources played no role in the study design; collection, analysis, and interpretation of data; writing of the report; or the decision to submit the article for publication.

\section{Disclosure}

Jiro Masuya has received personal compensation from Otsuka Pharmaceutical, Eli Lilly, Astellas, and Meiji Yasuda Mental Health Foundation, as well as grants from Pfizer. Masahiko Ichiki received personal compensation from Otsuka Pharmaceutical, Pfizer, Eli Lilly, Mitsubishi Tanabe Pharma, Mochida Pharmaceutical, Meiji Seika Pharma, Janssen Pharmaceutical, Takeda Pharmaceutical, MSD, Dainippon Sumitomo Pharma, and Eisai; grants from Otsuka Pharmaceutical, Eli Lilly, Eisai, Shionogi, Takeda Pharmaceutical, MSD, 
and Pfizer; and is a member of the advisory board of Meiji Seika Pharma. Ichiro Kusumi has received honoraria from Daiichi Sankyo, Dainippon Sumitomo Pharma, Eisai, Eli Lilly, Janssen Pharmaceutical, Lundbeck, Meiji Seika Pharma, Mochida Pharmaceutical, MSD, Mylan, Novartis Pharma, Ono Pharmaceutical, Otsuka Pharmaceutical, Pfizer, Shionogi, Shire, Taisho Toyama Pharmaceutical, Takeda Pharmaceutical, Tsumura, and Yoshitomiyakuhin, and has received research/grant support from Asahi Kasei Pharma, Astellas, Daiichi Sankyo, Dainippon Sumitomo Pharma, Eisai, Eli Lilly, Mochida Pharmaceutical, Novartis Pharma, Otsuka Pharmaceutical, Pfizer, Shionogi, Takeda Pharmaceutical and Tanabe Mitsubishi Pharma. Takeshi Inoue is a member of the advisory boards of Pfizer, Novartis Pharma, and Mitsubishi Tanabe Pharma and has received personal fees from Mochida Pharmaceutical, Takeda Pharmaceutical, Eli Lilly, Janssen Pharmaceutical, MSD, Taisho Toyama Pharmaceutical, Yoshitomiyakuhin, and Daiichi Sankyo; grants from Shionogi, Astellas, Tsumura, and Eisai; and grants and personal fees from Otsuka Pharmaceutical, Dainippon Sumitomo Pharma, Mitsubishi Tanabe Pharma, Kyowa Pharmaceutical Industry, Pfizer, Novartis Pharma, and Meiji Seika Pharma. Yota Fujimura received research and grant support from Novartis Pharma, Otsuka Pharmaceutical, Astellas, Dainippon Sumitomo Pharma, and Shionogi. Shinji Higashi received honoraria from Dainippon Sumitomo Pharma and Novartis Pharma. Kuniyoshi Toyoshima does not have any actual or potential conflict of interest.

\section{References}

1. World Health Organization. Definition of Health in Preamble to the Constitution of the World Health Organization. World Health Organization, Geneva, 2013. World Medical Association Declaration of Helsinki: ethical principles for medical research involving human subjects. JAMA. 1946;310:2191-2194. doi:10.1001/jama.2013.281053

2. Lindert J, Bain PA, Kubzansky LD, et al. Well-being measurement and the WHO health policy Health 2010: systematic review of measurement scales. Eur J Public Health. 2015;25:731-740. doi:10.1093/eur$\mathrm{pub} / \mathrm{cku} 193$

3. Howell RT, Kern ML, Lyubomirsky S. Health benefits: meta-analytically determining the impact of well-being on objective health outcomes. Health Psychol Rev. 2007;1:83-136. doi:10.1080/ 17437190701492486

4. Keyes CLM. The mental health continuum: from languishing to flourishing in life. J Health Soc Behav. 2002;43:207-222. doi:10.2307/ 3090197
5. Kanai Y, Takaesu Y, Nakai Y, et al. The influence of childhood abuse, adult life events, and affective temperaments on the well-being of the general, nonclinical adult population. Neuropsychiatr Dis Treat. 2016;12:823-832. doi:10.2147/NDT.S100474

6. Elwood LS, Wolitzky-Taylor K, Olatunji BO. Measurement of anxious traits: a contemporary review and synthesis. Anxiety Stress Coping. 2012;25:647-666. doi:10.1080/10615806.2011.582949

7. Weger M, Sandi C. High anxiety trait: a vulnerable phenotype for stress-induced depression. Neurosci Biobehav Rev. 2018;87:27-37. doi:10.1016/j.neubiorev.2018.01.012

8. Knowles KA, Olatunji BO. Specificity of trait anxiety in anxiety and depression: meta-analysis of the state-trait anxiety inventory. Clin Psychol Rev. 2020;82:101928. doi:10.1016/j.cpr.2020.101928

9. Basten U, Stelzel C, Fiebach CJ. Trait anxiety modulates the neural efficiency of inhibitory control. $J$ Cogn Neurosci. 2011;23:3132-3145. doi:10.1162/jocn_a_00003

10. Sylvester $\mathrm{CM}$, Corbetta M, Raichle $\overline{\mathrm{M}} \overline{\mathrm{E}}$, et al. Functional network dysfunction in anxiety and anxiety disorders. Trends Neurosci. 2012;35:527-535. doi:10.1016/j.tins.2012.04.012

11. Shimizu Y, Kitagawa N, Mitsui N, et al. Neurocognitive impairments and quality of life in unemployed patients with remitted major depressive disorder. Psychiatry Res. 2013;210:913-918. doi:10.1016/j.psychres.2013.08.030

12. Kaiser RH, Andrews-Hanna JR, Wager TD, et al. Large-scale network dysfunction in major depressive disorder: a meta-analysis of resting-state functional connectivity. JAMA Psychiatry. 2015;72:603-611. doi:10.1001/jamapsychiatry.2015.0071

13. Sumiyoshi T, Watanabe K, Noto $\mathrm{S}$, et al. Relationship of cognitive impairment with depressive symptoms and psychosocial function in patients with major depressive disorder: cross-sectional analysis of baseline data from PERFORM-J. J Affect Disord. 2019;258:172-178. doi:10.1016/j.jad.2019.07.064

14. Toyoshima $\mathrm{K}$, Inoue $\mathrm{T}$, Masuya $\mathrm{J}$, et al. Evaluation of subjective cognitive function using the Cognitive Complaints in Bipolar Disorder Rating Assessment (COBRA) in Japanese adults. Neuropsychiatr Dis Treat. 2019;15:2981-2990. doi:10.2147/NDT. S218382

15. Toyoshima K, Inoue T, Shimura A, et al. Associations between the depressive symptoms, subjective cognitive function, and presenteeism of Japanese adult workers: a cross-sectional survey study. BioPsychoSocial Med. 2020;14:10. doi:10.1186/s13030-020-00183-x

16. Rosa AR, Mercadé C, Sánchez-Moreno J, et al. Validity and reliability of a rating scale on subjective cognitive deficits in bipolar disorder (COBRA). J Affect Disord. 2013;150:29-36. doi:10.1016/j. jad.2013.02.022

17. van Rijsbergen MWA, Mark RE, Kop WJ, et al. Psychological factors and subjective cognitive complaints after stroke: beyond depression and anxiety. Neuropsychol Rehabil. 2019;29:1671-1684. doi:10.1080/09602011.2018.1441720

18. Wallis O, Bol Y, Köhler S, et al. Anxiety in multiple sclerosis is related to depressive symptoms and cognitive complaints. Acta Neurol Scand. 2020;141:212-218. doi:10.1111/ane.13191

19. Toyoshima K, Inoue T, Masuya J, et al. Associations among childhood parenting, affective temperaments, depressive symptoms, and cognitive complaints in adult community volunteers. J Affect Disord. 2020;276:361-368. doi:10.1016/j.jad.2020.07.107

20. Toyoshima K, Inoue T, Masuya J, et al. Does subjective cognitive function mediate the effect of affective temperaments on functional disability in Japanese adults? Neuropsychiatr Dis Treat. 2020;16:1675-1684. doi:10.2147/ndt.s256647

21. Toyoshima K, Inoue T, Masuya J, et al. Structural equation modeling approach to explore the influence of childhood maltreatment in adults. PLoS One. 2020;15:e239820. doi:10.1371/journal. pone. 0239820 
22. Spielberger CD. Manual for the State-Trait Anxiety Inventory STAI (Form Y). Palo Alto: Consulting Psychologists Press; 1983.

23. Hidano N, Fukuhara M, Iwawaki M, et al. State-Trait Anxiety Inventory-Form JYZ. Tokyo [in Japanese]: Japan UNI Agency; 2000.

24. Spitzer RL, Kroenke K, Williams JB. Validation and utility of a self-report version of PRIME-MD: the PHQ primary care study. Primary Care Evaluation of Mental Disorders. Patient Health Questionnaire. JAMA. 1999;282:1737-1744. doi:10.1001/ jama.282.18.1737

25. Kroenke K, Spitzer RL, Williams JB. The PHQ-9: validity of a brief depression severity measure. J Gen Intern Med. 2001;16:606-613. doi:10.1046/j.1525-1497.2001.016009606.x

26. Muramatsu K, Miyaoka H, Kamijima K, et al. The patient health questionnaire, Japanese version: validity according to the mini-international neuropsychiatric interview-plus. Psychol Rep. 2007;101:952-960. doi:10.2466/pr0.101.3.952-960

27. Muramatsu K, Miyaoka H, Kamijima K, et al. Performance of the Japanese version of the Patient Health Questionnaire-9 (J-PHQ-9) for depression in primary care. Gen Hosp Psychiatry. 2018;52:64-69. doi:10.1016/j.genhosppsych.2018.03.007

28. Toyoshima K, Fujii Y, Mitsui N, et al. Validity and reliability of the Cognitive Complaints in Bipolar Disorder Rating Assessment (COBRA) in Japanese patients with bipolar disorder. Psychiatry Res. 2017;254:85-89. doi:10.1016/j.psychres.2017.04.043

29. Miskowiak KW, Burdick KE, Martinez-Aran A, et al. Methodological recommendations for cognition trials in bipolar disorder by the International Society for Bipolar Disorders Targeting Cognition Task Force. Bipolar Disord. 2017;19:614-626. doi:10.1111/bdi.12534

30. Sell H, Nagpal R. Assessment of subjective well-being: the subjective well-being inventry (SUBI), in: Reg. Health Papers SEARO (New Delhi: World Health Organization Regional Office for South East Asia); 1992. Available from: https://apps.who.int/iris/handle/ 10665/204813. Accessed December 21, 2020.
31. tonan K, Sonoda A, Ono Y. Production of the subjective well-being inventory, Japanese ed.: it's reliability and validity. Jpn J Heal Psychol. 1995;8:12-19. doi:10.11560/jahp.8.2_12

32. Ono Y, Yoshimura K. The Subjective Well-Being Inventory Japanese Version. Tokyo: Kanekoshobo; 2001.

33. Topiwala A, Suri S, Allan C, et al. Subjective cognitive complaints given in questionnaire: relationship with brain structure, cognitive performance and self-reported depressive symptoms in a 25 -year retrospective cohort study. Am $J$ Geriatr Psychiatry. 2021;29:217-226. doi:10.1016/j.jagp.2020.07.002

34. Prado CE, Watt S, Treeby MS, Crowe SF. Performance on neuropsychological assessment and progression to dementia: a meta-analysis. Psychol Aging. 2019;34:954-977. doi:10.1037/ pag0000410

35. Zuniga KE, Mackenzie MJ, Kramer A, McAuley E. Subjective memory impairment and well-being in community-dwelling older adults. Psychogeriatrics. 2016;16:20-26. doi:10.1111/psyg.12112

36. Olsson LA, Hagnelius NO, Olsson H, Nilsson TK. Subjective well-being in Swedish active seniors or seniors with cognitive complaints and its relation to commonly available biomarkers. Arch Gerontol Geriatr. 2013;56(2):303-308. doi:10.1016/j. archger.2012.07.009

37. Murakoshi A, Mitsui N, Masuya J, et al. Personality traits mediate the association between perceived parental bonding and well-being in adult volunteers from the community. Biopsychosoc Med. 2020;14:28. doi:10.1186/s13030-020-00198-4

38. Bouldin ED, Taylor CA, Knapp KA, et al. Unmet needs for assistance related to subjective cognitive decline among community-dwelling middle-aged and older adults in the US: prevalence and impact on health-related quality of life. Int Psychogeriatr. 2020;1-14. doi:10.1017/S1041610220001635
Neuropsychiatric Disease and Treatment

\section{Publish your work in this journal}

Neuropsychiatric Disease and Treatment is an international, peerreviewed journal of clinical therapeutics and pharmacology focusing on concise rapid reporting of clinical or pre-clinical studies on a range of neuropsychiatric and neurological disorders. This journal is indexed on PubMed Central, the 'PsycINFO' database and CAS, and is the official journal of The International Neuropsychiatric Association (INA). The manuscript management system is completely online and includes a very quick and fair peer-review system, which is all easy to use. Visit http://www.dovepress.com/testimonials.php to read real quotes from published authors. 